\title{
Rectangular Meshes Construction of the Human Urethra Using 3-D GVF Snakes
}

\author{
Yu-Tai Ching, Chih-Yang Lin, Kheng-Chong Tan, Kiang-Long Lau, \\ Dept. of Computer and Information Science, National Chiao-Tung Univerity, Hsin-Chu 300, \\ Taiwan \\ C. H. Yang and C. A. Lin \\ Dept. of Power Mechanical Engineering, National Tsing-Hua University, Hsin-Chu 30013, Taiwan
}

\begin{abstract}
Accurate 3-D rectangular meshes construction of pipes is important and valuable for Engineering and Medicine to analyze the fluid mechanics. Hypertrophied prostate is suffered common by aged patient. Medicine trusts that the pathogenic reason can be interpreted using the statistics of analysis of fluid mechanics. We use serials of methods to construct the 3-D rectangular meshes of a patient urethra CT volumetric data given from medicine, and for mechanic engineering to gather statistics.
\end{abstract}

Keywords: Rectangular mesh, 3D geometric model construction, gradient vector flow, active contour, urodynamic, computational fluid dynamics.

\section{INTRODUCTION}

In this paper, serials of methods are proposed to half-automatically construct from binary raw volume to rectangular meshes. We use 3-D gradient vector flow (GVF) active contour modeling (snakes) to find the centerline out. An active contour algorithm is used to move the snake toward the centerline. We use GVF method to model the external force and ignore the internal force of snakes. Thus, we can use the snake to accurate decide the centerline of urethra. Afterwards, some linear transformation is used to find the orthogonal cross sections to tangent vector of the centerline. Furthermore, region growing method and alpha shape method are used to identify the contour on cross section. Finally, a brute forced intersections prevention algorithm and contours alignment method are proposed such we can construct rectangular meshes completely. Our method consists of four major processes: (1) GVF snake, (2) the orthogonal plane to the tangent of the centerline, (3) Alpha shapes, (4) assert clockwise direction for contour nodes, (5) intersection prevention, and (6) resample contour's nodes, and (7) contours alignment, which are detailed in the next section. Several experimental results are demonstrated in Section 3 to show the robustness of the proposed techniques.

\section{METHODS}

\section{$\underline{2.1 \text { Gradient Vector Flow Snake }}$}

$\mathrm{Xu}$ and Prince proposed gradient vector flow (GVF) to solve the first two problems encountered in the traditional active contour model. The method is a new class of external forces for active contour. A dense vector fields are derived from images by minimizing certain energy function. The minimization is achieved by solving a pair of decoupled linear partial differential equations that diffuse the gradient vectors of a gray-level or binary edge map computed from image. We call an active contour that uses gradient vector flow as its external force as a GVF snake. There are two main advantages of a GVF snake: The free initialization and good convergence to boundary concavities. The initialization can be inside, outside, or across an object's boundary.

After the GVF computation, we use greedy active contour algorithm to deform the snake, the estimated contour from previous step towards the centerline. The CEASAR centerline extraction algorithm let us know that GVF has good properties for finding centerline of pipes. We simply demonstrate that centerline can also be found using snake through 
the GVF properties. The large capture range of GVF and fastest greedy snake model are combined to make the finding easily.

Traditional snakes describe an energy minimization problem for finding the contour of a local feature in an image. As the snake seeks to minimize its overall energy, its shape will converge on the image gradient contour. Energy in this active contour model is represented by two force terms: $E_{i n t}, E_{\text {ext }}$. The total energy is therefore:

$$
E_{\text {snake }}=\int E_{\text {int }}(v(s))+E_{\text {ext }}(v(s)) d s,
$$

Due to GVF vector's properties, $V_{G}(x, y)=[u(x, y), \mathrm{v}(x, y)]$, that points to object boundary, where $(x, y)$ represents pixels coordinates and $u$ and $v$ are projections of a vector in $x$ and $y$ direction respectively on 2-D spaces. We extend the GVF greedy snake method proposed by Chuang and Lie to 3-D spaces. We assume that each voxel in the snake curve move from $P_{i, j}$ to $P_{i, j+1}$ where $i$ is voxel index in the snake curve and $j$ is the iteration number. The new model based on greedy search algorithm is defined to be:

$$
P_{i, j+1}=P_{i, j}+\left[V_{\text {int }}\left(P_{i, j}\right)+\Gamma V_{e x t}\left(P_{i, j}\right)\right]
$$

Where $V_{\text {int }}$ is internal force vector that minimizes the energy function:

$$
E\left(V_{\text {int }}\right)=a E_{\text {cont }}\left(V_{\text {int }}\right)+\beta E_{\text {curv }}\left(V_{\text {int }}\right)
$$

$E_{\text {cont }}$ and $E_{\text {curv }}$ are continuity and curvature forces, respectively. Due to discontinuities or bending, $V_{\text {ext }}$ is the external force vector that directs the points of curve to object boundary. Supposed 6-connected in 3-D space, we average the respectively GVF field to be the external force vector field. That is:

$$
\begin{gathered}
V_{e x t}(x, y, z)= \\
\frac{1}{6}\left[V_{G}(x-1, y, z)+V_{G}(x+1, y, z)+\right. \\
V_{G}(x, y-1, z)+V_{G}(x, y+1, z)+ \\
\left.V_{G}(x, y, z-1)+V_{G}(x, y, z+1)\right]
\end{gathered}
$$

There are a lot of coefficients, $a, \beta$ and $\Gamma$, in this scheme. According to Chuang and Lie's simulation, they told us that $a=\beta=0$ and $\Gamma=1$ are adopted. Such, the $V_{\text {int }}$ must equal to zero in equation (3). Concluded that, our model becomes to:

$$
P_{i, j+1}=P_{i, j} \dashv V_{e x t}\left(P_{i, j}\right) .
$$

It's no internal force and the deformation of snake entirely depends on GVF external force. According to the results of CEASAR algorithm, whatever 6/18/26 connected, the final result of centerline would be no difference.

When a snake deforms, the distances between vertices of the snake will change. This may result in local variation as well as in global changes in the resolution of the model. Both are undesirable, but slight local variation in the resolution is unavoidable. What we can do is to keep this variation between certain limits by periodically resample the snake along its path. We resample the snake according to a user defined parameter $l_{\text {des. }}$ Then we resample the snake to keep the distance between the neighboring vertices between $0.5 l_{\text {des }}$ and $1.5 l_{\text {des }}$. When the distance is less than $0.5 l_{\text {des }}$, we merge tow adjacent vertices into a single vertex. If the distance 
is longer than $1.5 l_{\mathrm{des}}$, we insert a vertex between the vertices.
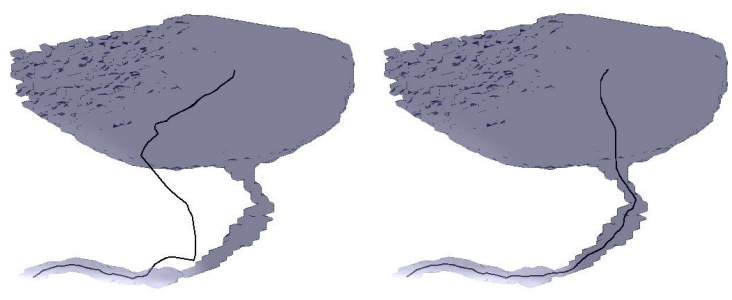

Fig. 1. Snake deformation

\subsection{The orthogonal plane to the tangent of the centerline}

The following step of our proposition is finding the orthogonal plane to the tangent of the centerline. After the centerline calculation, the tangent vector of each node in snake can be computed using differentiation of the centerline. We propose a fast and simple way to find the plane out with less matrix production.

$$
\left[\begin{array}{l}
x^{\prime} \\
y^{\prime} \\
z^{\prime}
\end{array}\right]=R_{y} R_{x} T\left[\begin{array}{l}
x \\
y \\
z
\end{array}\right],
$$

It's an old topic about 3-D transformation in computer graphics. If a fixed pointed and a vector be given, the origin of the new coordinate system moves to the fixed point and the new z-axis aligns to the vector direction. A transition and 2 rotations matrices production is shown in equation (6), where $T$ is a transition matrix, $R_{x}$ and $R_{y}$ are rotation matrices.

A set of $z^{\prime}=0$ in 3-D space is the plane members of we need. It can be translated to 2-D whether we ignore the zaxis. At first, bounding box of the plane is needed that we are just interesting in the urethral contour. For each new coordinate of $\left(x^{\prime}, y^{\prime}, 0\right)$ is 1-1 mapping to old coordinate of $(x, y, z)$.

$$
\left[\begin{array}{l}
x \\
y \\
z
\end{array}\right]=T^{-1} R_{x}^{-1} R_{y}^{-1}\left[\begin{array}{l}
x^{\prime} \\
y^{\prime} \\
0
\end{array}\right],
$$

A set of points in the plane can be found with a matrix production easily.

\section{$\underline{2.3 \text { Alpha Shapes }}$}

The concept of alpha-shapes developed by Edelsbrunner and Mücke (1994) formalizes the intuitive notion of "shape" for spatial point sets. The alpha-shape is a mathematically well-defined generalization of the convex hull and is a sub-graph of the Delaunay triangulation. Given a finite point set, a family of shapes can be derived from the Delaunay triangulation of the point set: a real parameter $a$ controls the desired level of detail.

We parameterize the $a$ value with 2.5, after the alpha-shapes computation, a contour round the set of points of urethra be derived. 


\section{$\underline{2.4 \text { Assert clockwise direction for contour nodes }}$}

Assertion of same direction of all contours is needed for correctness of contours alignment because the order of contour nodes derived from alpha-shapes does not keep the direction.
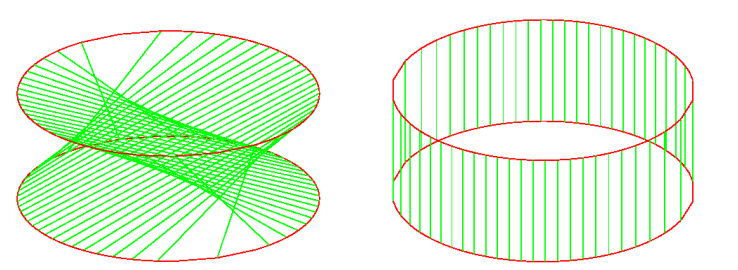

Fig. 2. Results of rectangular meshes compared between different direction contours.

\section{$\underline{2.5 \text { Intersection prevention }}$}

We propose a method of detecting and preventing the intersections between previous calculated plane and current calculated contour. Intersection prevention also needs for accuracy in contours alignment part.

We propose the intersection preventive algorithm as following:

Initial Less_Than_Zero, Same_Side as Boolean

Initial $D$ as floating point

Calculate $D$ of node to plane equation

Set Less_Than_Zero $=(D<0)$

For each contour's node

Begin

Calculate $D$ of node to plane equation

Set Same_Side $=(\mathrm{D}>0)$

If Less_Than_Zero not equal to Same_Side Intersection detected, discard contour

End $\{$ For $\}$

No Intersection be detected between contour and plane

Where plane equation is:

$$
A x \dashv B y \dashv C z \dashv D=0 .
$$

It's given $A, B$, and $C$ as normalized vector and a fixed point as $x, y$, and $z$. Uniqueness of $D$ is determined after substitution. If there exists two difference of signed value, positive and negative, of $\mathrm{D}$ in contour nodes, determined that intersection occurred. We just discard this contour.

\subsection{Resample contour's nodes}


After the intersection prevention part, two contours must be aligned. Assumed that all contours have same number of nodes, rectangular meshes can be aligned and connected between both contours. We divide all contours to 100 nodes.

\section{$\underline{2.7 \text { Contours alignment }}$}

The final step of rectangular meshes construction is contours alignment. An assumption is proposed that the rectangular meshes are constructed whether the summation of all edges connected between two contours must be minimized for non-twisted meshes. A brute force algorithm compares all alignment ordered summation that to select the minimized aligned order. It cost $O\left(n^{2}\right)$ time complexity of the comparisons.
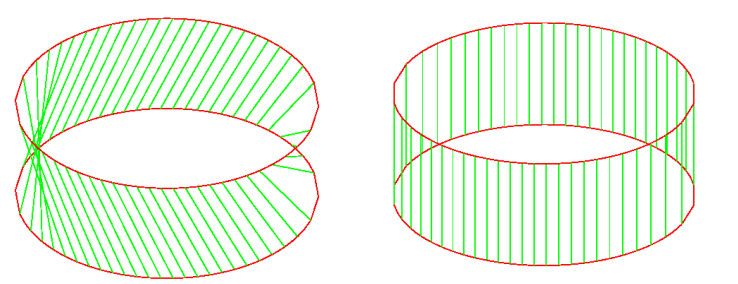

Fig. 3. Twisted and non-twisted alignment between two contours.

\section{RESULTS}

In this section, we present two results obtained by used of the proposed method. A sequence of urethral images from CT of 151 images with the size of 286x286 pixels per image is used in experiment. The final results of rectangular meshes with different views from fixed slice are shown as Figure. The proposed methods were implemented on a PC with a Pentium III $(800 \mathrm{MHz})$ CPU running on Windows 2000 operating system. The overall execution time took less than 2 minutes.

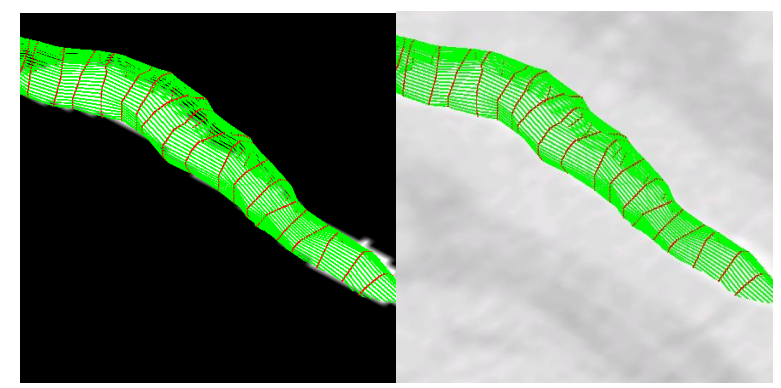

Figure 4: Fixed slice of 14 with binary and raw data
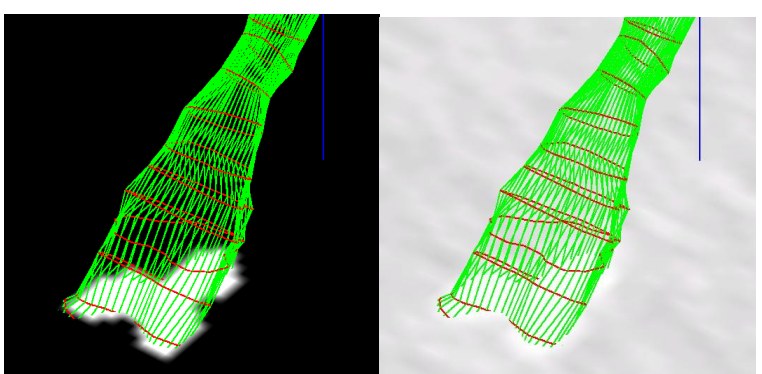

Fig. 5. Fixed slice of 53 with binary and raw data. 


\section{Governing equations}

The working fluid is assumed to be incompressible and Newtonian. The partial differential equations governing the conservation of mass and momentum are Reynolds averaged. In the present complex environment, the non-orthogonal curvilinear system is employed. The Cartesian coordinate and the curvilinear coordinate are $x_{i}(x, y, z)$ and $\xi_{i}(\xi, 1, \zeta)$, respectively. The transformed equations are expressed as follows:

\section{Continuity equation:}

$$
\frac{1}{J}\left[\frac{\partial U}{\partial \xi}+\frac{\partial V}{\partial \eta}+\frac{\partial W}{\partial \zeta}\right]=0
$$

\section{Momentium equation:}

$$
\begin{aligned}
& \frac{1}{J} \frac{\partial}{\partial \xi_{j}}\left[\rho U_{j} u_{i}\right]=-\frac{b_{i}^{j}}{J} \frac{\partial p}{\partial \xi_{j}}+\frac{1}{J} \frac{\partial}{\partial \xi_{j}}\left[\frac{\mu}{J}\left(b_{k}^{j} b_{i}^{l} \frac{\partial u_{k}}{\partial \xi_{l}}+B_{j}^{l} \frac{\partial u_{i}}{\partial \xi_{l}}\right)\right. \\
& \left.-\rho \overline{u_{i} u_{j}}\right]
\end{aligned}
$$

$\mathrm{U}, \mathrm{V}$ and $\mathrm{W}$ represent the contravariant velocity vector along the curvilinear coordinate $(\xi, \imath, \zeta)$ and are expressed as,

$$
\begin{aligned}
& U=\rho\left[b_{1}^{1} u+b_{2}^{1} v+b_{3}^{1} w\right] \\
& V=\rho\left[b_{1}^{2} u+b_{2}^{2} v+b_{3}^{2} w\right] \\
& W=\rho\left[b_{1}^{3} u+b_{2}^{3} v+b_{3}^{3} w\right]
\end{aligned}
$$

where $\mathrm{u}, \mathrm{v}, \mathrm{w}$ represent Cartesian velocities, and the coefficients are as follows:

$$
\begin{array}{ccc}
b_{1}^{1}=y_{\eta} z_{\zeta}-y_{\zeta} z_{\eta} & b_{1}^{2}=y_{\zeta} z_{\xi}-y_{\xi} z_{\zeta} & b_{1}^{3}=y_{\xi} z_{\eta}-y_{\eta} z_{\xi} \\
b_{2}^{1}=x_{\zeta} z_{\eta}-x_{\eta} z_{\zeta} & b_{2}^{2}=x_{\xi} z_{\zeta}-x_{\zeta} z_{\xi} & b_{2}^{3}=z_{\xi} x_{\eta}-x_{\xi} z_{\eta} \\
b_{3}^{1}=x_{\eta} y_{\zeta}-x_{\zeta} y_{\eta} & b_{3}^{2}=y_{\xi} x_{\zeta}-x_{\xi} y_{\zeta} & b_{3}^{3}=x_{\xi} y_{\eta}-y_{\xi} x_{\eta}
\end{array}
$$

For laminar flow, the Reynolds stresses are zero. As for turbulent flow, within the framework of eddy-viscosity and adopting Boussinesq approximation, the Reynolds stresses are approximated as,

$$
-\rho \overline{u_{i} u_{j}}=-\frac{2}{3} \delta_{j}^{i} \rho k+\frac{\mu_{t}}{J}\left(b_{k}^{j} b_{i}^{l} \frac{\partial u_{k}}{\partial \xi_{l}}+B_{j}^{l} \frac{\partial u_{i}}{\partial \xi_{l}}\right)
$$

For turbulent flow simulations, the standard $k-\varepsilon$ model proposed by Spalding and Launder (1974) is adopted, i.e.

$$
\mu_{t}=\rho C_{\mu} \frac{k^{2}}{\varepsilon}
$$

The governing equations forkandeare, $\kappa$ equation:

$$
\begin{aligned}
& \frac{1}{J} \frac{\partial}{\partial \xi_{j}}\left[\rho U_{j} k\right]=\frac{1}{J} \frac{\partial}{\partial \xi_{j}}\left[\frac{1}{J}\left(\mu+\frac{\mu_{t}}{\sigma_{k}}\right) B_{j}^{k} \frac{\partial k}{\partial \xi_{k}}\right] \\
& +G_{k}-\rho \varepsilon
\end{aligned}
$$

E equation:

$$
\begin{aligned}
& \frac{1}{J} \frac{\partial}{\partial \xi_{j}}\left[\rho U_{j} \varepsilon\right]=\frac{1}{J} \frac{\partial}{\partial \xi_{j}}\left[\frac{1}{J}\left(\mu+\frac{\mu_{t}}{\sigma_{\varepsilon}}\right) B_{j}^{k} \frac{\partial \varepsilon}{\partial \xi_{k}}\right] \\
& +\frac{\varepsilon}{k}\left[C_{\varepsilon 1} G-C_{\varepsilon 2} \rho \varepsilon\right]
\end{aligned}
$$




$$
G_{k}=\mu_{t} \frac{1}{J^{2}}\left[b_{j}^{l} \frac{\partial u_{i}}{\partial \xi_{l}}+b_{i}^{l} \frac{\partial u_{j}}{\partial \xi_{l}}\right] b_{j}^{l} \frac{\partial u_{i}}{\partial \xi_{l}}
$$

where the coefficients can be found in Spalding and Launder (1974).

\section{Test Geometry}

As mentioned earlier, one of the goals is to identify the major cause of the pressure drop in the urethral flow field. Therefore, the flow rate, minimum obstructed area and obstructed shape are used as the parameters to help to clarify the results. The success of the predictive methodology depends on how faithfully the mathematical model reflects the actual anatomy and physiology of the individual and on how efficiently the simulations can be performed. Based on the non-invasive computer tomography (CT) images (Figure 6), a three-dimensional lower urethral model can be constructed. (Figure 7).
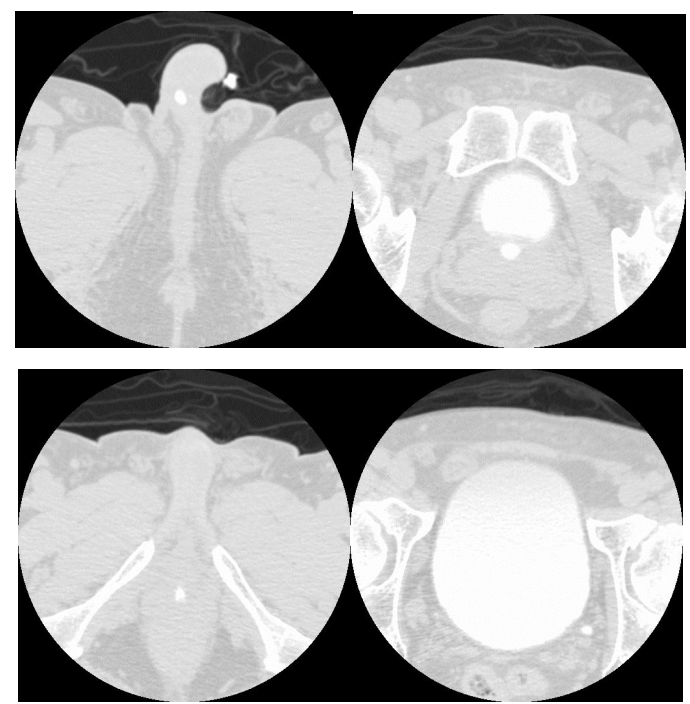

Fig. 6: CT images.

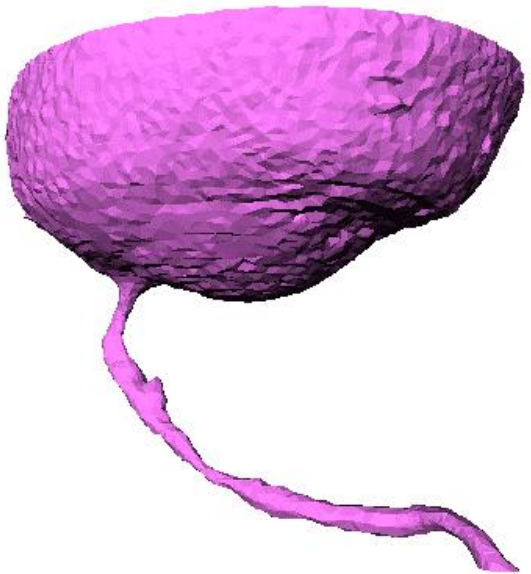

Fig. 7. Test geometry. 


\section{Numerical Procedure}

The present numerical procedure solves discretised versions of all equations over a collocated finite-volume arrangement. The principle of mass-flux continuity is imposed indirectly via the solution of pressure-correction equations according to the SIMPLE algorithm. The flow-property values at volume faces contained in the convective fluxes which arise from the finite-volume integration process are approximated by the hybrid scheme. The computed solution is assumed to have converged to its steady-state when the magnitude of the absolute residual sources of mass and momentum, normalised by the respective inlet fluxes, falls below $0.01 \%$.

\section{Results}

The influence of the flow rates on the pressure losses is shown in Figure 8. It can be observed that the pressure loss exists mainly in the obstructed zone. In the obstructed cases, there are two major losses. One is the major or frictional loss, and the other is the minor loss. Minor loss happens due to the change of geometry. Figures 9a to 9d show the pressure contours at four cross-section planes along the longitudinal direction. The variation of the pressure in each cross section is small compared to the variations at different longitudinal locations. At the obstructed cross section (Figure 9c), the pressure reaches a minimum due to the acceleration of the fluid. Excessive pressure loss occurs near this area. The detailed pressure profiles can assist the urologist to diagnose patients having bladder outlet obstruction symptoms.

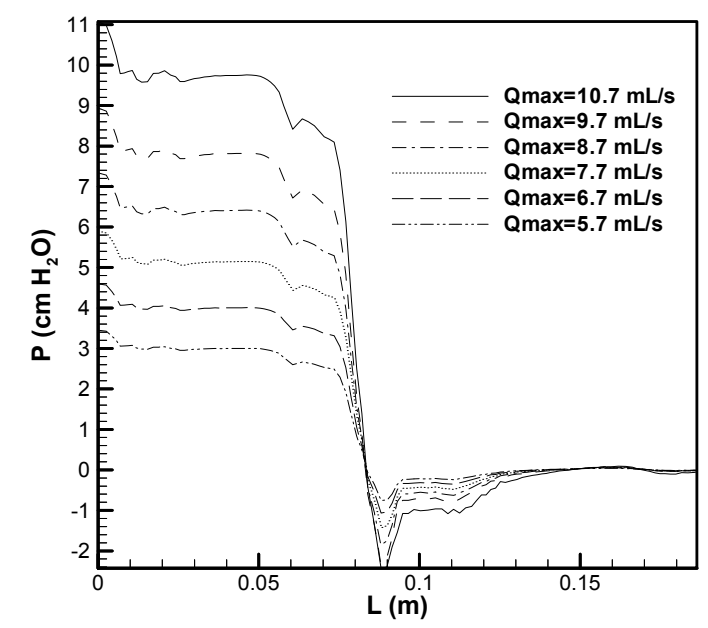

Fig.8. Pressure distribution in different flow rates 


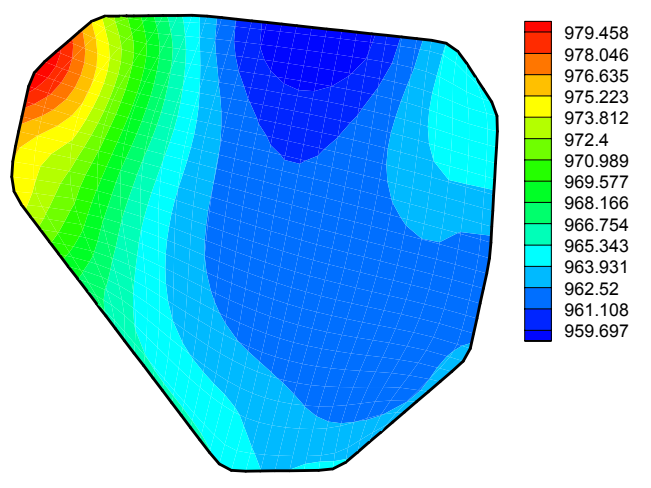

(a)

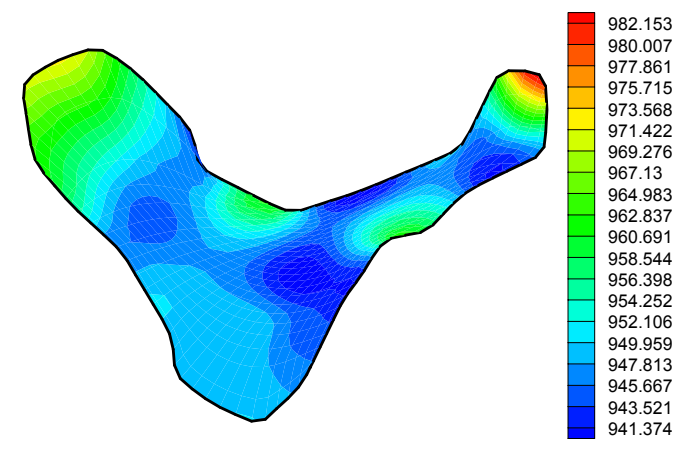

(b) 


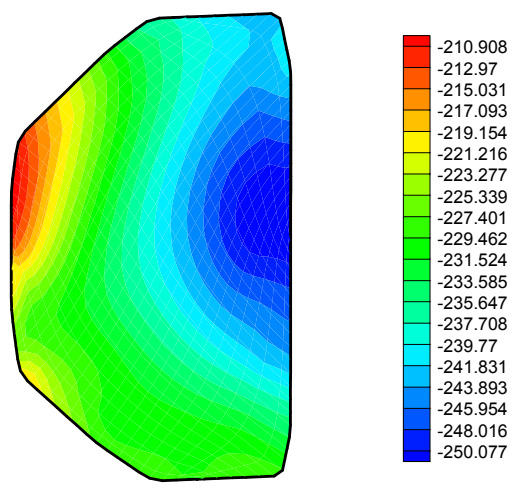

(c)

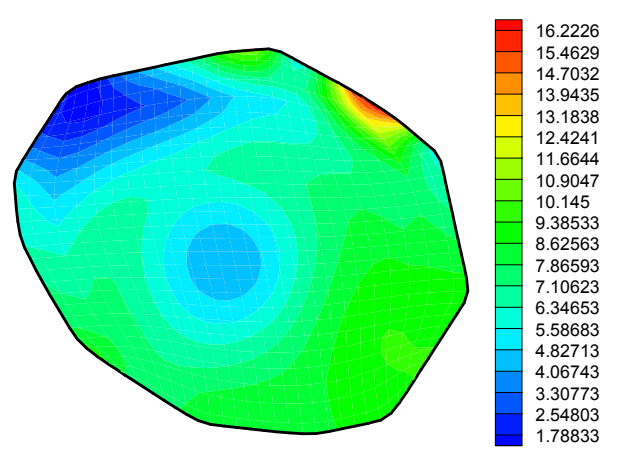

(d)

Fig. 9. (a) Pressure contours at the inlet near the bladder (contour unit in Pascal). (b) Pressure contours at the middle of the prostate (contour unit in Pascal). (c) Pressure contours at the obstructed cross-section near the end of the prostate (contour unit in Pascal). (d) Pressure contours at the exit (contour unit in Pascal).

\section{REFERENCES}

1. C. Xu, J.L. Prince, “Gradient Vector Flow: A new external force for snake, " IEEE Proc, Conf. On Comput. Vis Patt. Recog. (CVPR) 1997, pp. 66-77.

2. C. Xu, J.L. Prince, "Snakes, shapes, and Gradient Vector Flow, " IEEE Trans, on Image Process. (March 1998) 359-369.

3. M. Kass, A. Witkin, and D. Terzopoulox, "Snakes: Active Contour Models, " Int. J. Comput. Vis, vol. 1, pp. 321$331,1987$.

4. D. J. Williams and M. Shah, "A fast algorithm for active contours and curvature estimation, " CVGIP: Image Understanding, Vol. 55, No. 1, pp. 14-26, 1992. 
5. S. Lobregt and M. A. Viergever, “A discrete dynamic contour model, ” IEEE Trans. Med. Imag. Vol. 14, pp. 12-24, Mar. 1995.

6. C. H. Chuang and W. N. Lie, "Fast and Accurate Active Contours for Object Boundary Segmentation," Circuits and Systems, 2000. IEEE APCCAS 2000. pp. 473-476

7. I. Bitter, M. Sato, M. Bender, K. T. McDonnell, A. Kaufman and Ming Wan, "CEASAR: A Smooth, Accurate and Robust Centerline Extraction Algorithm, " Visualization 2000. Proceedings, 2000, pp. 45-52, 539. 